\title{
RESIDUAL PROPERTIES OF SIMPLE GRAPHS
}

\author{
BELINDA TROTTA
}

(Received 6 November 2009)

\begin{abstract}
Clark et al. ['The axiomatizability of topological prevarieties', Adv. Math. 218 (2008), 1604-1653] have shown that, for $k \geq 2$, there exists a Boolean topological graph that is $k$-colourable but not topologically $k$-colourable; that is, for every $\epsilon>0$, it cannot be coloured by a paintbrush of width $\epsilon$. We generalize this result to show that, for $k \geq 2$, there is a Boolean topological graph that is 2-colourable but not topologically $k$-colourable. This graph is an inverse limit of finite graphs which are shown to exist by an Erdős-style probabilistic argument of Hell and Nešetřil ['The core of a graph', Discrete Math. 109 (1992), 117-126]. We use the fact that there exists a Boolean topological graph that is 2-colourable but not $k$-colourable, and some other results (some new and some previously known), to answer the question of which finitely generated topological residual classes of graphs are axiomatizable by universal Horn sentences. A more general version of this question was raised in the above-mentioned paper by Clark et al., and has been investigated by various authors for other structures.
\end{abstract}

2000 Mathematics subject classification: primary 05C80; secondary 08C15, 05C15, 57M15.

Keywords and phrases: random graph, graph colouring, Boolean topological graph, universal Horn class, axiomatization.

\section{Introduction}

Let $\mathbf{C}$ be the topological graph shown in Figure 1, where the points of the topological space are the vertices, and the topology is that inherited from $\mathbb{R}^{2}$. (This is a modified version of the graph in Figure 4 of Clark et al. [5].)

Clearly $\mathbf{C}$ is 2-colourable; indeed it is a disjoint union of two paths. However, $\mathbf{C}$ is not topologically 2-colourable, that is, for each $\epsilon>0$, the graph $\mathbf{C}$ cannot be 2-coloured by a paintbrush of width $\epsilon$; equivalently, there is no continuous homomorphism from $\mathbf{C}$ to $\mathbf{K}_{2}$, where $\mathbf{K}_{2}$ is the complete graph on two vertices endowed with the discrete topology. To see that $\mathbf{C}$ is not topologically 2-colourable, note that $a$ and $b$ must be different colours, and therefore by backwards induction $a_{1}$ and $b_{1}$ are different colours, which is impossible since both are related to $c$. Theorem 7.5 of [5] shows that an analogous graph also exists for $k \geq 3$; indeed, for every $k \geq 2$, there is a topological graph whose topology is Boolean (that is, compact,

This article is largely reproduced from the author's PhD Thesis, Axiomatisability of Topological Prevarieties, La Trobe University, 2009.

(C) 2010 Australian Mathematical Publishing Association Inc. 0004-9727/2010 \$16.00 


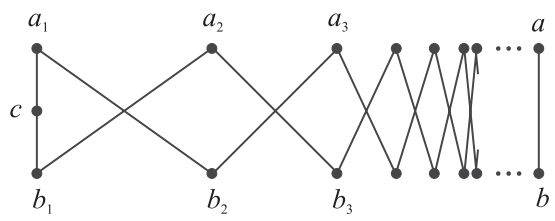

FIGURE 1. A topological graph that is 2-colourable but not topologically 2-colourable.

totally disconnected, with topologically closed edge relation) that is $k$-colourable but not topologically $k$-colourable.

We will generalize this result and show that for all $k \geq 2$, there is a Boolean topological graph that is 2 -colourable but not topologically $k$-colourable. The proof is not constructive: the required graph is an inverse limit of finite graphs, each of which is shown to exist by a probabilistic argument from Hell and Nešetřil [10] (based on an argument of Erdős). However, it is possible to give a constructive proof of a related result; see Section 4.

The notion of (topological) $k$-colourability is in fact a particularization of a more general idea, that of being (topologically) residually a subgraph of another graph. Nešetřil and Pultr [13] showed that for each $k$, there is a graph $\mathbf{C}_{k}$ such that a graph $\mathbf{G}$ is $k$-colourable if and only if it is residually a subgraph of $\mathbf{C}_{k}$. Moreover, it is shown in [5] that if $\mathbf{G}$ is a Boolean topological graph, then $\mathbf{G}$ is topologically $k$-colourable if and only if it is topologically residually a subgraph of $\mathbf{C}_{k}$. (Throughout this paper, we will use 'subgraph' to mean 'induced subgraph', except where we make it clear that we are using another definition.)

Let $\mathbf{G}$ be a finite graph with the discrete topology. The graph in Figure 1 and the result of Nešetríl and Pultr (and its topological analogue) demonstrate that the following property does not always hold:

(*) every Boolean topological graph that is residually a subgraph of $\mathbf{G}$ is also topologically residually a subgraph of $\mathbf{G}$.

In this paper, we characterize the finite graphs $\mathbf{G}$ for which $(*)$ holds.

It turns out that the property $(*)$ is related to the axiomatizability by first-order sentences of the class $\mathcal{R}_{\mathrm{CT}}(\mathbb{S}(\mathbf{G}))$ of compact graphs that are topologically residually subgraphs of $\mathbf{G}$. Indeed, (*) holds if and only if $\mathcal{R}_{\mathrm{CT}}(\mathbb{S}(\mathbf{G}))$ is axiomatizable among Boolean topological graphs by a certain type of first-order sentences, called universal Horn $(\mathrm{uH})$ sentences. In this case we say that $\mathcal{R}_{\mathrm{CT}}(\mathbb{S}(\mathbf{G}))$ is $u H$ axiomatizable. We will characterize the simple graphs $\mathbf{G}$ for which $\mathcal{R}_{\mathrm{CT}}(\mathbb{S}(\mathbf{G}))$ is $\mathrm{uH}$ axiomatizable. We also consider a weaker version of $\mathrm{uH}$ axiomatizability. If $\mathcal{R}_{\mathrm{CT}}(\mathbb{S}(\mathbf{G}))$ is axiomatizable (among Boolean topological graphs) by a set of first-order sentences, we say it is firstorder axiomatizable. We will give results about first-order axiomatizability for simple graphs in a few specific cases.

The concept of $\mathrm{uH}$ axiomatizability for classes $\mathcal{R}_{\mathrm{CT}}(\mathbb{S}(\mathbf{X}))$, where $\mathbf{X}$ is a finite structure, was introduced by Clark et al. [4], who called it standardness. The theory of standardness has been applied in duality theory (see Davey and Talukder [7] and 
Davey [6]). Also, connections between standardness and other areas of algebra have been explored. Clark et al. [3] showed how standardness is related to certain properties of the syntactic congruences of an algebra. Jackson [12] discusses the relationship between residual bounds and standardness for Boolean topological algebras, in particular semigroups and groups.

\section{Preliminaries}

A simple graph $\mathbf{G}=\langle G ; \sim\rangle$ is a structure with a single symmetric and anti-reflexive binary relation $\sim$. We will just write 'graph' to mean 'simple graph', and we will use the same notation for graphs, or classes of graphs, and their corresponding topological versions. Much of the following discussion can be generalized to arbitrary structures with operations and relations; see [5].

Let $\mathcal{C}$ be a class of graphs and $\mathbf{G}$ a graph. We say that $\mathbf{G}$ is residually in $\mathcal{C}$ if there exists a homomorphism from $\mathbf{G}$ to some member of $\mathcal{C}$; and, for all $a, b \in \mathbf{G}$ with $a \neq b$ or $a \nsim b$, there exists $\mathbf{H} \in \mathcal{C}$ and a surjective homomorphism $\phi: \mathbf{G} \rightarrow \mathbf{H}$ such that $\phi(a) \neq \phi(b)$ or $\phi(a) \nsim \phi(b)$, respectively. (The first part of the condition ensures that the one-element looped graph is not in $\mathcal{R}(\mathcal{C})$, which simplifies the statements of our later results.) We will denote the class of graphs residually in $\mathcal{C}$ by $\mathcal{R}(\mathcal{C})$. If $\mathcal{C}$ consists of topological graphs, and $\mathbf{G}$ is also a topological graph, we say that $\mathbf{G}$ is topologically residually in $\mathrm{C}$ if the above condition holds and the homomorphisms can be chosen to be continuous. We write $\mathcal{R}_{\mathrm{CT}}(\mathcal{C})$ for the class of compact topological graphs topologically residually in $\mathcal{U}$. We write $\mathbb{S}(\mathcal{C})$ (or just $\mathbb{S}(\mathbf{G})$ if $\mathcal{C}=\{\mathbf{G}\}$ ) to denote the class of (induced) subgraphs of members of $\mathcal{C}$. It is well known that, for any class $\mathcal{C}$ of structures, $\mathcal{R}(\mathbb{S}(\mathcal{C}))$ consists of the structures isomorphic to a substructure of a direct product (with nonempty index set) of members of $\mathcal{C}$, and that $\mathcal{R}_{\mathrm{CT}}(\mathbb{S}(\mathcal{C})$ ) is the class of topological structures isomorphic to a topologically closed substructure of a direct product (with nonempty index set) of members of $\mathcal{C}$, where the direct product has the usual product topology.

A universal Horn sentence is a universally quantified first-order sentence of the form

$$
\bigvee_{i \in I} \neg \alpha_{i} \quad \text { or } \quad\left(\bigwedge_{i \in I} \alpha_{i}\right) \longrightarrow \beta
$$

where $I$ is finite (and possibly empty) and the $\alpha_{i}$ and $\beta$ are statements of the form $a \approx b$ or $a \sim b$. A universal Horn class is one that can be axiomatized by universal Horn sentences. If $\mathbf{G}$ is a finite graph, then $\mathcal{R}(\mathbb{S}(\mathbf{G}))$ is a $\mathrm{uH}$ class; indeed it is exactly the class of models of the universal Horn theory of $\mathbf{G}$, that is, we have $\mathcal{R}(\mathbb{S}(\mathbf{G}))=\operatorname{Mod}\left(\operatorname{Th}_{\mathrm{uH}}(\mathbf{G})\right)$ (see Burris and Sankappanavar [1, Theorem V.2.23]). A $\mathrm{uH}$ class $\mathcal{U}$ is finitely generated if $\mathcal{U}=\mathcal{R}(\mathbb{S}(\mathcal{C})$ ) for some finite class of finite graphs $\mathcal{C}$. Note that if $\mathcal{U}$ is a $\mathrm{uH}$ class, then $\mathbb{S}(\mathcal{U})=\mathcal{U}=\mathcal{R}(\mathcal{U})$.

Caicedo [2] gives the following classification and description of finitely generated $\mathrm{uH}$ classes of graphs, using results of Nešetřil and Pultr [13]. Throughout this paper, $\mathbf{S}_{n}$ denotes the $n$-element path and $\mathbf{A}_{n}$ denotes the $n$-element antichain. 
THEOREM 2.1. Let $\mathrm{U}$ be a finitely generated $\mathrm{uH}$ class of graphs. Then exactly one of the following is true:

(1) $\quad U=\mathcal{R}\left(\mathbb{S}\left(\mathbf{A}_{1}\right)\right)$;

(2) $\quad U=\mathcal{R}\left(\mathbb{S}\left(\mathbf{A}_{2}\right)\right)$;

(3) $\quad U=\mathcal{R}\left(\mathbb{S}\left(\mathbf{S}_{2}\right)\right)$;

(4) $\quad \mathcal{U}=\mathcal{R}\left(\mathbb{S}\left(\mathbf{S}_{3}\right)\right)$;

(5) $\quad \mathcal{R} \mathcal{R}\left(\mathbb{S}\left(\mathbf{S}_{4}\right)\right)$.

The class $\mathcal{R}\left(\mathbb{S}\left(\mathbf{S}_{3}\right)\right)$ is the uH class of all disjoint unions of complete bipartite graphs, and $\mathcal{R}\left(\mathbb{S}\left(\mathbf{S}_{4}\right)\right)$ is the uH class of all bipartite graphs. Furthermore, $\mathcal{U}$ is finitely axiomatizable by $u H$ sentences if and only if it is one of the types (1)-(4).

Recall from the introduction that a Boolean topological graph is a graph with a compact, totally disconnected topology where the edge relation is a closed subset of $\mathbf{G} \times \mathbf{G}$. If $\mathcal{U}$ is a class of graphs, we write $\mathcal{U}_{\mathrm{Bt}}$ to denote the class of Boolean topological graphs whose underlying graph is in $\mathcal{U}$, and $\mathcal{U}_{\text {fin }}$ for the class of finite members of $\mathcal{U}$, where each has the discrete topology. We will call $\mathcal{R}_{\mathrm{CT}}\left(\mathcal{U}_{\mathrm{fin}}\right)$ a topological residual class. When $\mathcal{U}$ is the $\mathrm{uH}$ class generated by a finite graph $\mathbf{G}$, we have $\mathcal{R}_{\mathrm{CT}}\left(\mathcal{U}_{\text {fin }}\right)=\mathcal{R}_{\mathrm{CT}}(\mathbb{S}(\mathbf{G}))$, and this is called the topological residual class generated by $\mathbf{G}$. We now give more precise statements of two theorems mentioned in the introduction.

Theorem 2.2 [13, Example 2.9(4)]. Let $k \in \mathbb{N}$. There exists a finite graph $\mathbf{C}_{k}$ such that a graph $\mathbf{G}$ is $k$-colourable if and only if $\mathbf{G} \in \mathcal{R}\left(\mathbb{S}\left(\mathbf{C}_{k}\right)\right)$.

Theorem 2.3 [5, Proposition 7.2]. Let $k \in \mathbb{N}$. Then a Boolean topological graph $\mathbf{G}$ is topologically $k$-colourable if and only if $\mathbf{G} \in \mathcal{R}_{\mathrm{CT}}\left(\mathbb{S}\left(\mathbf{C}_{k}\right)\right)$, where $\mathbf{C}_{k}$ is as in Theorem 2.2.

If $\Sigma$ is a set of sentences, we write $\operatorname{Mod}_{\mathrm{Bt}}(\Sigma)$ to denote the set of Boolean topological models of $\Sigma$. Recall from the introduction that $\mathcal{R}_{\mathrm{CT}}(\mathbb{S}(\mathbf{G}))$ is $\mathrm{uH}$ axiomatizable if and only if there exists a set $\Sigma$ of uH sentences such that $\mathcal{R}_{\mathrm{CT}}(\mathbb{S}(\mathbf{G}))=\operatorname{Mod}_{\mathrm{Bt}}(\Sigma)$. Since taking direct products and substructures preserves $\mathrm{uH}$ sentences, $\mathrm{uH}$ axiomatizability of $\mathcal{R}_{\mathrm{CT}}(\mathbb{S}(\mathbf{G}))$ is equivalent to the property that $\mathcal{R}_{\mathrm{CT}}(\mathbb{S}(\mathbf{G}))=\operatorname{Mod}_{\mathrm{Bt}}\left(\operatorname{Th}_{\mathrm{uH}}(\mathbf{G})\right)$. If $\mathcal{U}$ is a uH class, we say that $\mathcal{R}_{\mathrm{CT}}\left(\mathcal{U}_{\mathrm{fin}}\right)$ is $\mathrm{uH}$ axiomatizable if it is the class of Boolean topological models of some set of $\mathrm{uH}$ sentences. Using the fact that $\mathcal{U}$ is locally finite, it can be shown that $\mathcal{R}_{\mathrm{CT}}\left(\mathcal{U}_{\text {fin }}\right)$ is $\mathrm{uH}$ axiomatizable if and only if $\mathcal{R}_{\mathrm{CT}}\left(\mathcal{U}_{\text {fin }}\right)=\mathfrak{U}_{\mathrm{Bt}}$.

As noted in the introduction, for finite structures $\mathbf{X}, \mathrm{uH}$ axiomatizability of $\mathcal{R}_{\mathrm{CT}}(\mathbb{S}(\mathbf{X}))$ is equivalent to standardness, as defined in [4]. Clark et al. [5] extend the definition of standardness to include classes of the form $\mathcal{R}_{\mathrm{CT}}\left(\mathcal{U}_{\mathrm{fin}}\right)$ where $\mathcal{U}$ is a $\mathrm{uH}$ class of structures. They define $\mathcal{U}$ to be standard if $\mathcal{R}_{\mathrm{CT}}\left(\mathcal{U}_{\mathrm{fin}}\right)=\mathcal{U}_{\mathrm{Bt}}$. If $\mathcal{U}$ is locally finite (in particular, if $\mathcal{U}$ consists of relational structures or $\mathcal{U}$ is finitely generated), then $\mathcal{R}_{\mathrm{CT}}\left(\mathcal{U}_{\text {fin }}\right)$ is standard if and only if it is $\mathrm{uH}$ axiomatizable, but in general standardness is stronger than uH axiomatizability; see [5, Example 2.7]. 
In this paper we classify the $\mathrm{uH}$ axiomatizability of $\mathcal{R}_{\mathrm{CT}}\left(\mathcal{U}_{\mathrm{fin}}\right)$ for finitely generated $\mathrm{uH}$ classes $\mathcal{U}$ of simple graphs. The topological residual classes generated by $\mathbf{A}_{1}$ and $\mathbf{A}_{2}$ are obviously $\mathrm{uH}$ axiomatizable. We will show that $\mathcal{R}_{\mathrm{CT}}\left(\mathbb{S}\left(\mathbf{S}_{2}\right)\right)$ is also $\mathrm{uH}$ axiomatizable. We give a constructive inverse limit proof that $\mathcal{U}=\mathcal{R}\left(\mathbb{S}\left(\mathbf{S}_{3}\right)\right)$ is not $\mathrm{uH}$ axiomatizable. Finally, for $\mathcal{U}$ containing $\mathbf{S}_{4}$, we show that $\mathcal{R}_{\mathrm{CT}}\left(\mathcal{U}_{\text {fin }}\right)$ is not $\mathrm{uH}$ axiomatizable by applying an inverse limit technique from [5] to a probabilistic construction from [10]. This gives the following characterization of $\mathrm{uH}$ axiomatizability for simple graphs.

THEOREM 2.4. Let $\mathcal{U}$ be a finitely generated $u H$ class of graphs. Then $\mathcal{R}_{\mathrm{CT}}\left(\mathcal{U}_{\mathrm{fin}}\right)$ is $u$ H axiomatizable if and only if:

(1) $\quad \mathcal{U}=\mathcal{R}\left(\mathbb{S}\left(\mathbf{A}_{1}\right)\right)$; or

(2) $\quad \mathcal{U}=\mathcal{R}\left(\mathbb{S}\left(\mathbf{A}_{2}\right)\right)$; or

(3) $\mathcal{U}=\mathcal{R}\left(\mathbb{S}\left(\mathbf{S}_{2}\right)\right)$.

We will mainly consider uH axiomatizability for finitely generated $\mathrm{uH}$ classes of simple graphs; however, it has also been studied more generally. For example, if $\mathcal{U}$ is the $\mathrm{uH}$ class of all simple graphs (which is not finitely generated), then $\mathcal{R}_{\mathrm{CT}}\left(\mathcal{U}_{\text {fin }}\right)$ is $\mathrm{uH}$ axiomatizable (see [5, Theorem 7.3]); that is, $\mathcal{R}_{\mathrm{CT}}\left(\mathcal{U}_{\mathrm{fin}}\right)$ is exactly the class of Boolean topological simple graphs. Stralka [14] showed that the class of Priestly spaces, $\mathcal{R}_{\mathrm{CT}}\left(\mathcal{P}_{\text {fin }}\right)$, where $\mathcal{P}$ is the class of reflexive, anti-symmetric, transitive directed graphs, is not $\mathrm{uH}$ axiomatizable. In $[15,16]$, the author characterizes $\mathrm{uH}$ axiomatizability for some topological residual classes of directed graphs with certain properties. In particular, [16] contains a generalization of Stralka's result which characterizes uH axiomatizability for all topological residual classes of reflexive antisymmetric digraphs. Other generalizations of Stralka's example can be found in [5, Section 6]. UH axiomatizability, and the related concept of standardness, have also been studied for structures with operations, partial operations and relations; see [3-5] for many examples.

The following generalization of $\mathrm{uH}$ axiomatizability was introduced in [5]. Let $\mathcal{U}$ be a $\mathrm{uH}$ class. We say that $\mathcal{R}_{\mathrm{CT}}\left(\mathcal{U}_{\mathrm{fin}}\right)$ is first-order axiomatizable if every Boolean topological model of $\mathrm{Th}\left(\mathcal{R}_{\mathrm{CT}}\left(\mathcal{U}_{\text {fin }}\right)\right)$ is in $\mathcal{R}_{\mathrm{CT}}\left(\mathcal{U}_{\text {fin }}\right)$, that is, if $\mathcal{R}_{\mathrm{CT}}\left(\mathcal{U}_{\text {fin }}\right)$ is precisely the class of Boolean topological models of some set $\Sigma$ of first-order sentences. Obviously, every $\mathrm{uH}$ axiomatizable topological residual class is also first-order axiomatizable.

We do not have a characterization of first-order axiomatizability for finitely generated topological residual classes of graphs; however, some results are known. For example, [5, Theorem 7.6] shows that the class of topologically $k$-colourable graphs is not first-order axiomatizable. In Section 3, we will show that the topological residual class generated by $\mathbf{S}_{3}$ is not first-order axiomatizable. In Section 4 we consider, for $k \geq 3$, the class of Boolean topological graphs with the property of free topological $k$-colourability, a strengthening of topological $k$-colourability. We show that this class is not first-order axiomatizable. 
There are no known counter-examples to the conjecture that for simple graphs (or indeed, directed graphs in general), $\mathrm{uH}$ axiomatizability is equivalent to first-order axiomatizability. However, this is not true for arbitrary structures; see [5, Example 4.3] for an example of a finite lattice generating a topological residual class that is firstorder axiomatizable but not $\mathrm{uH}$ axiomatizable.

\section{Universal Horn axiomatizability for finitely generated uH classes}

In this section we prove Theorem 2.4. We also show that $\mathcal{R}_{\mathrm{CT}}\left(\mathbb{S}\left(\mathbf{S}_{3}\right)\right)$ is not firstorder axiomatizable.

We start by showing that $\mathcal{R}_{\mathrm{CT}}\left(\mathbb{S}\left(\mathbf{S}_{2}\right)\right)$ is $\mathrm{uH}$ axiomatizable. The following theorem is proved by Edwards [8, Ch. 5, Claim 6]; we give a similar proof here. If $\mathbf{G}$ is a graph and $X \subseteq G$, we say that $\mathbf{X}$ is independent if $(X \times X) \cap \sim=\varnothing$. Also we define $R(X):=\{y \in G \mid(\exists x \in X) x \sim y\}$. Note that if $X$ is closed, then so is $R(X)$.

THEOREM 3.1. The class $\mathcal{R}_{\mathrm{CT}}\left(\mathbb{S}\left(\mathbf{S}_{2}\right)\right)$ is uH axiomatizable.

PROOF. Let $\mathbf{G}$ be a topological graph in $\operatorname{Mod}_{\mathrm{Bt}}\left(\operatorname{Th}_{\mathrm{uH}}\left(\mathbf{S}_{2}\right)\right)$. We label the vertices of $\mathbf{S}_{2}$ by 0,1 . We first prove the following claim.

Claim. If $A$ and $B$ are disjoint closed independent subsets of $\mathbf{G}$, then there exists a continuous homomorphism $\phi: \mathbf{G} \rightarrow \mathbf{S}_{2}$ such that $\phi(A)=\{0\}$ and $\phi(B)=\{1\}$.

Now we will prove the claim. Since $A$ is closed and $(A \times A) \cap \sim=\varnothing$, there exists a clopen set $U_{0} \supseteq A$ such that $\left(U_{0} \times U_{0}\right) \cap \sim=\varnothing$ (that is, $U_{0}$ is independent), and $U_{0} \cap B=\varnothing$. Similarly, there exists a clopen independent set $V_{0} \supseteq B$ such that $V_{0} \cap U_{0}=\varnothing$. For each $x \in G \backslash\left(U_{0} \cup V_{0}\right)$, let $W_{x} \subseteq G \backslash\left(U_{0} \cup V_{0}\right)$ be a clopen independent set. Now $\left\{W_{x} \mid x \in G \backslash\left(U_{0} \cup V_{0}\right)\right\}$ is an open cover of $G \backslash\left(U_{0} \cup V_{0}\right)$, so there exists a finite subcover, $\left\{W_{0}, \ldots, W_{n}\right\}$, for some $n$. Assume that $i \geq 0$ and we have defined disjoint clopen independent sets $U_{i}, V_{i}$ such that $A \subseteq U_{i}$ and $B \subseteq V_{i}$, and $U_{i} \cup V_{i} \supseteq \bigcup_{k=0}^{i} W_{i}$. We define $U_{i+1}, V_{i+1}$ with the same properties. Since $\mathbf{G}$ contains no path of length greater than 1 and $U_{i}, V_{i}$ are disjoint, the sets $W_{i+1} \cap R\left(U_{i}\right)$ and $W_{i+1} \cap R\left(V_{i}\right)$ are disjoint. Therefore there exists a clopen set $Y \subseteq W_{i+1}$ containing $W_{i+1} \cap R\left(U_{i}\right)$ and disjoint from $W_{i+1} \cap R\left(V_{i}\right)$. Let $U_{i+1}=U_{i} \cup\left(W_{i+1} \backslash Y\right)$ and $V_{i+1}=V_{i} \cup Y$. It is easy to see that $U_{i+1}$ and $V_{i+1}$ have the required properties. Now let $U=U_{n}$ and $V=V_{n}$, so that $U$ and $V$ are independent and $\{U, V\}$ is a clopen partition of $\mathbf{G}$. Let $\phi: \mathbf{G} \rightarrow \mathbf{S}_{2}$ be given by $\phi(x)=0$ if $x \in U$ and $\phi(x)=1$ otherwise. This proves the claim.

Let $x, y \in \mathbf{G}$ with $x \neq y$. Then since $\{x\}$ and $\{y\}$ are closed independent sets, by the claim there exists a map $\phi: \mathbf{G} \rightarrow \mathbf{S}_{2}$ with $\phi(x)=0$ and $\phi(y)=1$. Now let $x, y \in \mathbf{G}$ with $x \nsim y$. Then $\{x, y\}$ is a closed independent set. Therefore by the claim there exists a continuous homomorphism $\phi: \mathbf{G} \rightarrow \mathbf{S}_{2}$ with $\phi(x)=\phi(y)=0$, so $\phi(x) \nsim \phi(y)$.

To show that $\mathcal{R}_{\mathrm{CT}}(\mathbb{S}(\mathbf{3}))$ is not $\mathrm{uH}$ axiomatizable, we use a theorem from [5] that gives a way of constructing Boolean topological graphs, as inverse limits, to witness 
non-uH-axiomatizability. We will now give a brief outline of the theory of inverse systems of graphs and state the theorem from [5] that we will use. A more detailed and general discussion of inverse limits can be found in [5].

An inverse system over $\mathbb{N}$ of topological graphs is a set $\left\{\mathbf{X}_{n} \mid n \in \mathbb{N}\right\}$ of topological graphs and a set of continuous connecting homomorphisms $\phi_{n}: \mathbf{X}_{n+1} \rightarrow \mathbf{X}_{n}$. The inverse limit of the system is the set

$$
\left\{x \in \prod_{n \in \mathbb{N}} \mathbf{X}_{n} \mid(\forall n) \phi_{n}\left(x_{n+1}\right)=x_{n}\right\},
$$

and is denoted $\lim _{\{}\left\{\mathbf{X}_{n} \mid n \in \mathbb{N}\right\}$. If each $\mathbf{X}_{n}$ is a finite nonempty graph (with the discrete topology), then the inverse limit is nonempty, and is a Boolean topological graph.

The following theorem is a simplified version of Theorem 3.5 in [5], and is stated here only for the case of graphs. We say that an inverse limit $\mathbf{X}=\lim \left\{\mathbf{X}_{n} \mid n \in \mathbb{N}\right\}$ is pointwise nonseparable with respect to a $\mathrm{uH}$ class $\mathcal{U}$ if there exists $r \in\{\sim,=\}$ and $a, b \in \mathbf{X}$ such that $(a, b) \notin r$ but, for all $n \in \mathbb{N}$, every $\mathbf{G} \in \mathcal{U}_{\text {fin }}$ and every homomorphism $\psi: \mathbf{X}_{n} \rightarrow \mathbf{G}$, we have $\left(\psi\left(a_{n}\right), \psi\left(b_{n}\right)\right) \in r$.

Theorem 3.2 [5, Theorem 3.5]. Let $\mathcal{U}$ be a uH class of graphs, and let $\mathbf{X}=$ $\underset{\lim }{\longleftarrow}\left\{\mathbf{X}_{n} \mid n \in \mathbb{N}\right\}$ be an inverse limit of finite graphs with surjective connecting maps.

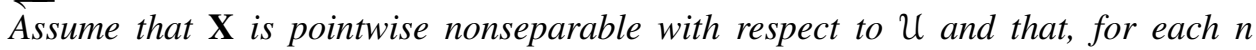
and each $Y \subseteq X_{n+1}$ such that $\phi_{n} \uparrow_{Y}$ is one-to-one, the graph $\mathbf{Y}$ is in $\mathcal{U}_{\mathrm{fin}}$. Then $\mathbf{X} \in \mathcal{U}_{\mathrm{Bt}} \backslash \mathcal{R}_{\mathrm{CT}}\left(\mathcal{U}_{\mathrm{fin}}\right)$, so $\mathcal{R}_{\mathrm{CT}}\left(\mathcal{U}_{\mathrm{fin}}\right)$ is not $u$ H axiomatizable.

We will use another theorem from [5] to show that the topological graph that we construct to witness non-uH-axiomatizability also witnesses non-first-order axiomatizability. The statement below is a simplified version that applies to graphs.

TheOREM 3.3 [5, Theorem 5.2]. Let $\mathcal{U}$ be a uH class of graphs and suppose that $\mathbf{X}$ is a topological graph witnessing the non-uH-axiomatizability of $\mathcal{R}_{\mathrm{CT}}\left(\mathcal{U}_{\mathrm{fin}}\right)$ (that is, $\left.\mathbf{X} \in \mathcal{U}_{\mathrm{Bt}} \backslash \mathcal{R}_{\mathrm{CT}}\left(\mathcal{U}_{\mathrm{fin}}\right)\right)$. If, up to isomorphism, there are only finitely many different connected components in $\mathbf{X}$, and they are all finite, then $\mathcal{R}_{\mathrm{CT}}\left(\mathcal{U}_{\mathrm{fin}}\right)$ is not first-order axiomatizable.

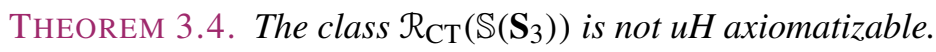

Proof. For $n \in \mathbb{N}$ let $\mathbf{T}_{n}$ be the $\left(3^{n}+1\right)$-element path with vertices labelled $1,2, \ldots, 3^{n+1}$. Let $\phi_{n}: \mathbf{T}_{n+1} \rightarrow \mathbf{T}_{n}$ be given by $\phi_{n}(x)=q_{x}+r_{x}$, where $q_{x}$ and $r_{x}$ are, respectively, the quotient and remainder when $x$ is divided by 3 . Figure 2 shows $\phi_{1}$ and $\phi_{2}$. It is obvious from the picture (and easy to check) that the connecting maps are homomorphisms.

For each $n$, we have $1 \nsim 3^{n}+1$ in $\mathbf{T}_{n}$. Let $\psi: \mathbf{T}_{n} \rightarrow \mathbf{S}_{3}$ be a homomorphism. Note that $\mathbf{S}_{3}$ satisfies the $\mathrm{uH}$ formula $x_{1} \sim x_{2} \sim x_{3} \sim x_{4} \longrightarrow x_{1} \sim x_{4}$, so $\psi\left(\mathbf{T}_{n}\right)$ also satisfies this $\mathrm{uH}$ formula. So $\psi(1) \sim \psi(4)$, and if $\psi(1) \sim \psi(2 k)$ for some $k$, then

$$
\psi(1) \sim \psi(2 k) \sim \psi(2 k+1) \sim \psi(2 k+2)=\psi(2(k+1)),
$$



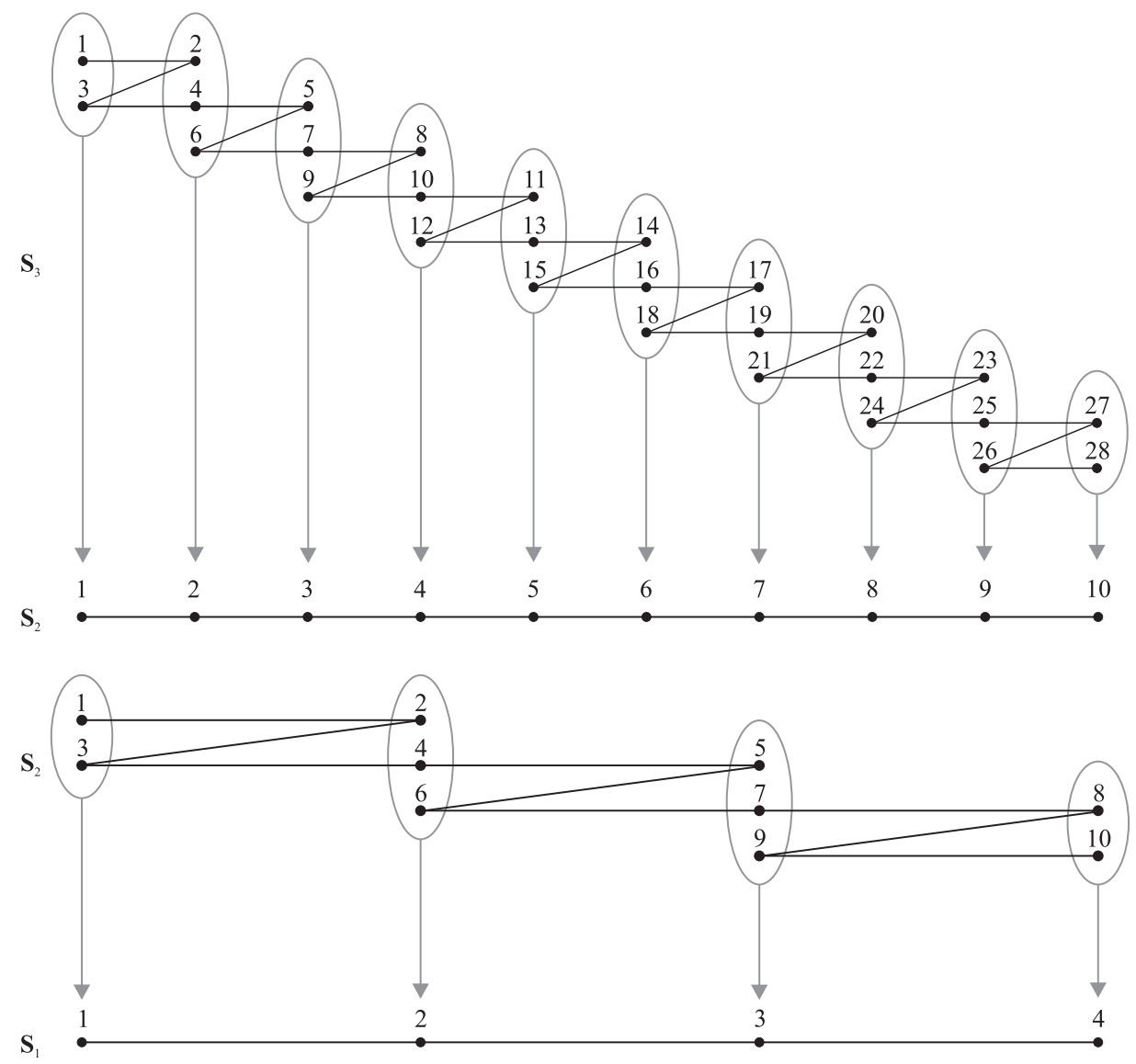

FIGURE 2. The maps $\phi_{1}$ and $\phi_{2}$ (indicated by grey arrows).

so that $\psi(1) \sim \psi(2(k+1))$. Therefore by induction we have $\psi(1) \sim \psi(2 k)$ for each $k \geq 2$, and in particular $\psi(1) \sim \psi\left(3^{n}+1\right)$. So $\mathbf{T}:=\lim _{\longleftarrow}\left\{\mathbf{T}_{n} \mid n \in \mathbb{N}\right\}$ is pointwise nonseparable with respect to $\mathcal{R}\left(\mathbb{S}\left(\mathbf{S}_{3}\right)\right)$.

Now let $Y \subseteq T_{n+1}$ and suppose that $\mathbf{Y}$ contains a path of more than three elements, that is, there exists $i$ such that $i, i+1, i+2, i+3 \in Y$. We show that $\phi_{n}: \mathbf{T}_{n+1} \rightarrow \mathbf{T}_{n}$ is not one-to-one on $\mathbf{Y}$. If $r_{i}=0$, then

$$
\phi_{n}(i+1)=q_{i+1}+r_{i+1}=q_{i}+1=q_{i+3}+r_{i+3}=\phi_{n}(i+3) ;
$$

if $r_{i}=1$, then

$$
\phi_{n}(i)=q_{i}+r_{i}=q_{i}+1=q_{i+2}+r_{i+2}=\phi_{n}(i+2) ;
$$

and if $r_{i}=2$, then

$$
\phi_{n}(i)=q_{i}+r_{i}=q_{i}+2=q_{i+2}+r_{i+2}=\phi_{n}(i+2) .
$$


Therefore, if $\phi_{n}$ is one-to-one on $\mathbf{Y}$, then $\mathbf{Y}$ contains no path of more than three elements, that is, $\mathbf{Y}$ is a disjoint union of isolated vertices, and paths with two or three elements. Thus $\mathbf{Y}$ is a disjoint union of complete bipartite graphs and is therefore in $\mathcal{R}\left(\mathbb{S}\left(\mathbf{S}_{3}\right)\right)$, by Theorem 2.1. Theorem 3.2 now shows that $\mathcal{R}_{\mathrm{CT}}\left(\mathbb{S}\left(\mathbf{S}_{3}\right)\right)$ is not uH axiomatizable.

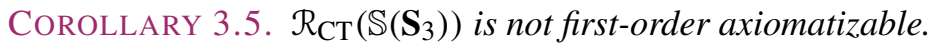

Proof. Let $\mathbf{X}$ be the inverse limit constructed in the proof of Theorem 3.4. Suppose that $\mathbf{X}$ contains a path with more than three elements, so that there exist distinct elements $a, b, c, d$ of $\mathbf{X}$ with $a \sim b \sim c \sim d$. Then there exists $n$ such that, for $m \geq n$, we have $a_{m}, b_{m}, c_{m}, d_{m}$ all distinct. In particular, $a_{n+1}, b_{n+1}, c_{n+1}, d_{n+1}$ are all distinct, and $a_{n+1} \sim b_{n+1} \sim c_{n+1} \sim d_{n+1}$. But then, by the argument in the proof of Theorem 3.1, we have $\left|\phi_{n}\left(\left\{a_{n+1}, b_{n+1}, c_{n+1}, d_{n+1}\right\}\right)\right|<4$, a contradiction. So $\mathbf{X}$ contains no path with more than three elements. Also, the degree of each vertex in $\mathbf{X}$ is at most two, since this is true for every graph $\mathbf{T}_{n}$. Therefore, up to isomorphism, there are only finitely many connected components in $\mathbf{X}$ and they are all finite, so by Theorem 3.3 the class $\mathcal{R}_{\mathrm{CT}}\left(\mathbb{S}\left(\mathbf{S}_{3}\right)\right)$ is not first-order axiomatizable.

We now show that if $\mathcal{U}$ is a $\mathrm{uH}$ class of graphs that has a bound on the chromatic number of its members and contains the $\mathrm{uH}$ class of all bipartite graphs, the class $\mathcal{R}_{\mathrm{CT}}\left(\mathcal{U}_{\text {fin }}\right)$ is not $\mathrm{uH}$ axiomatizable. In particular, this means that for every finitely generated $\mathrm{uH}$ class $\mathcal{U}$ of graphs containing the $\mathrm{uH}$ class of all bipartite graphs, $\mathcal{R}_{\mathrm{CT}}\left(\mathcal{U}_{\text {fin }}\right)$ is not $\mathrm{uH}$ axiomatizable. Again, we use an inverse limit technique from [5]. The following theorem is a restatement of Theorem 3.9 from [5] restricted to the case of graphs.

TheOREM 3.6. Let $\mathcal{U}, \mathcal{V}, \mathcal{W}$ be $u H$ classes of graphs with $\mathcal{V} \subseteq \mathcal{U} \subseteq \mathcal{W}$, and $\mathbf{X}=$ $\lim \left\{\mathbf{X}_{n} \mid n \in \mathbb{N}\right\}$ an inverse limit of finite graphs, where the connecting maps are

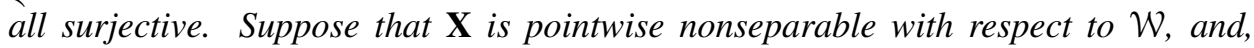
for all $n$, every subgraph of $\mathbf{X}_{n}$ with at most $n$ elements is a member of $\mathcal{V}$. Then $\mathbf{X} \in \mathcal{V}_{\mathrm{Bt}} \backslash \mathcal{R}_{\mathrm{CT}}\left(\mathcal{W}_{\mathrm{fin}}\right)$, so $\mathcal{R}_{\mathrm{CT}}\left(\mathcal{U}_{\mathrm{fin}}\right)$ is not uH axiomatizable.

Note that it suffices to find an inverse system of finite graphs $\left\{\mathbf{X}_{n} \mid n \in \mathbb{N}\right\}$ with surjective connecting maps, such that the inverse limit is pointwise nonseparable with respect to $\mathcal{W}$, and, for each $n$, each proper subgraph of $\mathbf{X}_{n}$ is in $\mathcal{V}$.

The following result of Hell and Nešetřil [10] implies the existence of an inverse limit with the required properties. A similar result, applying to structures with finitely many relations of finite arity, is proved in Feder and Vardi [9, Theorem 5]. Also, an inverse system with the required properties can be constructed from Theorem 2.3 of Hodkinson and Venema [11]; however, the Hell and Nešetřil construction is slightly simpler, and gives a more interesting result from the point of view of this paper; the inverse limit is a forest rather than just bipartite.

Throughout this paper, we use $\mathbf{K}_{k}$ to denote the complete graph with $k$ elements, labelled $1,2, \ldots, k$. 
TheOREM 3.7 [10, Theorem 4]. Let $n \in \mathbb{N}$, and let $\mathbf{G}$ and $\mathbf{G}^{\prime}$ be finite graphs such that there is no homomorphism from $\mathbf{G}$ to $\mathbf{G}^{\prime}$. Then there is a finite graph $\mathbf{H}$ such that $\mathbf{H}$ contains no cycles of length less than n, and there is a homomorphism from $\mathbf{H}$ to $\mathbf{G}$ but no homomorphism from $\mathbf{H}$ to $\mathbf{G}^{\prime}$.

COROLlaRY 3.8. Let $k \geq 2$. There exists an inverse system over $\mathbb{N}$, with surjective connecting maps, of finite graphs $\mathbf{H}_{n}$ such that, for each $n$, the chromatic number of $\mathbf{H}_{n}$ is at least $k+1$ and $\mathbf{H}_{n}$ has no cycles of length less than $n+1$.

PROOF. We construct the digraphs $\mathbf{H}_{n}$ inductively. Let $\mathbf{H}_{1}$ be the graph $\mathbf{K}_{k+1}$. Now suppose that we have constructed $\mathbf{H}_{n}$ with the required properties, so that there is no homomorphism from $\mathbf{H}_{n}$ to $\mathbf{K}_{k}$. By Theorem 3.7 there exists a graph $\mathbf{H}_{n+1}$ and a surjective connecting homomorphism from $\mathbf{H}_{n+1}$ to $\mathbf{H}_{n}$ such that $\mathbf{H}_{n+1}$ has no cycles of length less than $n+2$, and there are no homomorphisms from $\mathbf{H}_{n+1}$ to $\mathbf{K}_{k}$, that is, $\mathbf{H}_{n+1}$ has chromatic number at least $k+1$.

THEOREM 3.9. Let $k \geq 2$. Then there exists a Boolean topological graph that is 2-colourable, but not topologically $k$-colourable. Therefore:

(1) if $\mathcal{U}$ is a $u H$ class of graphs such that all graphs in $\mathcal{U}$ are $k$-colourable, and $\mathcal{U}$ contains the class of all bipartite graphs, then $\mathcal{R}_{\mathrm{CT}}\left(\mathcal{U}_{\mathrm{fin}}\right)$ is not $u H$ axiomatizable;

(2) if $\mathcal{U}$ is a finitely generated $u H$ class containing the $u H$ class of all bipartite graphs, then $\mathcal{R}_{\mathrm{CT}}\left(\mathcal{U}_{\mathrm{fin}}\right)$ is not $u$ H axiomatizable.

PROOF. We prove the first statement; the others will follow easily. Let $\mathcal{V}$ be the class of 2-colourable graphs, and let $\mathcal{W}$ be the class of $k$-colourable graphs. Both are $\mathrm{uH}$ classes by Theorem 2.2. By Corollary 3.8, there exists an inverse system $\left\{\mathbf{H}_{n} \mid n \in \mathbb{N}\right\}$ of graphs with surjective connecting homomorphisms $\phi_{n}: \mathbf{H}_{n+1} \rightarrow \mathbf{H}_{n}$ such that each $n$-element subgraph of $\mathbf{H}_{n}$ is bipartite (indeed, it is a forest) and therefore in $\mathcal{V}$, but $\mathbf{H}_{n}$ has chromatic number at least $k+1$. For each $n$, there are no homomorphisms from $\mathbf{H}_{n}$ to $\mathbf{K}_{k}$, where $\mathbf{K}_{k}$ is the complete graph with $k$ elements, and therefore no homomorphisms from $\mathbf{H}_{n}$ to $\mathbf{G}$ for $\mathbf{G} \in \mathcal{W}$. So the inverse limit $\mathbf{H}$ is (vacuously) pointwise nonseparable with respect to $\mathcal{W}$. Thus, by Theorem 3.6, we have $\mathbf{H} \in \mathcal{V}_{\mathrm{Bt}} \backslash \mathcal{R}_{\mathrm{CT}}\left(\mathcal{W}_{\text {fin }}\right)$. By Theorem 2.3, $\mathcal{R}_{\mathrm{CT}}\left(\mathcal{W}_{\text {fin }}\right)$ is the class of topologically $k$-colourable graphs, so that $\mathbf{H}$ is 2 -colourable but not topologically $k$-colourable. To prove (1), note that since $\mathcal{V} \subseteq \mathcal{U} \subseteq \mathcal{W}$, the class $\mathcal{R}_{\mathrm{CT}}\left(\mathcal{U}_{\text {fin }}\right)$ is not uH axiomatizable. Finally, (2) is a special case of (1).

We can now prove Theorem 2.4.

Proof of Theorem 2.4. Let $\mathcal{U}$ be a finitely generated $u$ H class. If $\mathcal{U}$ is $\mathcal{R}\left(\mathbb{S}\left(\mathbf{A}_{1}\right)\right)$ or $\mathcal{R}\left(\mathbb{S}\left(\mathbf{A}_{2}\right)\right)$, then it is trivial to prove that $\mathcal{R}_{\mathrm{CT}}\left(\mathcal{U}_{\mathrm{fin}}\right)$ is uH axiomatizable. Theorem 3.1 shows that $\mathcal{R}\left(\mathbb{S}\left(\mathbf{S}_{2}\right)\right)$ is also $\mathrm{uH}$ axiomatizable. For the converse, suppose that $\mathcal{U}$ is not one of $\mathcal{R}\left(\mathbb{S}\left(\mathbf{A}_{1}\right)\right), \mathcal{R}\left(\mathbb{S}\left(\mathbf{A}_{2}\right)\right)$ or $\mathcal{R}\left(\mathbb{S}\left(\mathbf{S}_{2}\right)\right)$. Then by Theorem $2.1, \mathcal{U}$ is either the class $\mathcal{R}\left(\mathbb{S}\left(\mathbf{S}_{3}\right)\right)$, or $\mathcal{U}$ contains the class of all bipartite graphs. Therefore Theorem 3.4 or Theorem 3.9 shows that $\mathcal{R}_{\mathrm{CT}}\left(\mathcal{U}_{\text {fin }}\right)$ is not $\mathrm{uH}$ axiomatizable. 


\section{Freely $k$-colourable graphs}

We say that a graph $\mathbf{G}$ is freely $k$-colourable if there exists a homomorphism from $\mathbf{G}$ to $\mathbf{K}_{k}$ (that is, $\mathbf{G}$ is $k$-colourable) and, for each pair $u, v \in \mathbf{G}$ with $u \nsim v$ and $u \neq v$, there exist homomorphisms $\phi_{1}, \phi_{2}: \mathbf{G} \rightarrow \mathbf{K}_{k}$ such that $\phi_{1}(u)=\phi_{1}(v)$ and $\phi_{2}(u) \neq \phi_{2}(v)$ (that is, the pair $u, v$ may be coloured the same or differently). We say that a topological graph is freely topologically $k$-colourable if it is freely $k$-colourable and the homomorphisms witnessing this can be chosen to be continuous. We will show that the class of Boolean topological graphs that are freely topologically $k$-colourable is not first-order axiomatizable.

LEMMA 4.1. A graph $\mathbf{G}$ is freely $k$-colourable if and only if $\mathbf{G} \in \mathcal{R}\left(\mathbb{S}\left(\mathbf{K}_{k}\right)\right)$.

Proof. First let $\mathbf{G}$ be freely $k$-colourable. Let $u, v \in \mathbf{G}$ with $u \neq v$. If $u \sim v$, then since $\mathbf{G}$ is $k$-colourable, there is a homomorphism $\phi: \mathbf{G} \rightarrow \mathbf{K}_{k}$, and $\phi(u) \neq \phi(v)$. If $u \nsim v$ then since $\mathbf{G}$ is freely $k$-colourable there is a homomorphism $\phi: \mathbf{G} \rightarrow \mathbf{K}_{k}$ with $\phi(u) \neq \phi(v)$. Now let $u, v \in G$ with $u \nsim v$. If $u \neq v$, then since $\mathbf{G}$ is freely $k$ colourable, there is a homomorphism $\phi: \mathbf{G} \rightarrow \mathbf{K}_{k}$ with $\phi(u)=\phi(v)$, so $\phi(u) \nsim \phi(v)$. In the case where $u=v$, every homomorphism $\phi: \mathbf{G} \rightarrow \mathbf{K}_{k}$ has $\phi(u) \nsim \phi(v)$.

Suppose that $\mathbf{G} \in \mathcal{R}\left(\mathbb{S}\left(\mathbf{K}_{k}\right)\right)$. Clearly $\mathbf{G}$ is $k$-colourable. Let $u, v \in \mathbf{G}$ with $u \nsim v$ and $u \neq v$. Since $u \neq v$ there is a homomorphism $\phi: \mathbf{G} \rightarrow \mathbf{K}_{k}$ with $\phi(u) \neq \phi(v)$. Since $u \nsim v$, there is a homomorphism $\phi: \mathbf{G} \rightarrow \mathbf{K}_{k}$ with $\phi(u) \nsim \phi(v)$, which means that $\phi(u)=\phi(v)$ as $\mathbf{K}_{k}$ is a complete graph.

LEMMA 4.2. A Boolean topological graph $\mathbf{G}$ is freely topologically $k$-colourable if and only if $\mathbf{G} \in \mathcal{R}_{\mathrm{CT}}\left(\mathbb{S}\left(\mathbf{K}_{k}\right)\right)$.

PROOF. The argument is the same as that in the proof of Lemma 4.1, except that the homomorphisms are replaced by continuous homomorphisms.

Let $k \geq 3$. We will prove that $\mathcal{R}_{\mathrm{CT}}\left(\mathbf{K}_{k}\right)$ is not $\mathrm{uH}$ axiomatizable. Since $\mathcal{R}\left(\mathbb{S}\left(\mathbf{K}_{k}\right)\right)$ contains the class of all bipartite graphs, this result is a consequence of Theorem 3.9. However, in this particular case, we can give a nonprobabilistic construction of a topological graph witnessing non-uH-axiomatizability. Furthermore, we will also be able to use this construction to prove that $\mathcal{R}_{\mathrm{CT}}\left(\mathbb{S}\left(\mathbf{K}_{k}\right)\right)$ is not first-order axiomatizable, using another technique from [5], Theorem 4.6 below.

We begin by showing that $\mathcal{R}_{\mathrm{CT}}\left(\mathbb{S}\left(\mathbf{K}_{3}\right)\right)$ is not $\mathrm{uH}$ axiomatizable, and the proof for larger $k$ will proceed by induction. The proof uses Theorem 3.6.

LEMMA 4.3. $\mathcal{R}_{\mathrm{CT}}\left(\mathbb{S}\left(\mathbf{K}_{3}\right)\right)$ is not uH axiomatizable.

PROOF. For $n \geq 3$, let $\mathbf{G}_{n}$ be the graph with elements

$$
\left\{a, b, a_{1}, \ldots, a_{n}, b_{1}, \ldots, b_{n-1}\right\}
$$

and the relation $\sim$ where:

- $\quad a \sim a_{i}$, for all $i$

- $a_{1} \sim a_{n}$ 


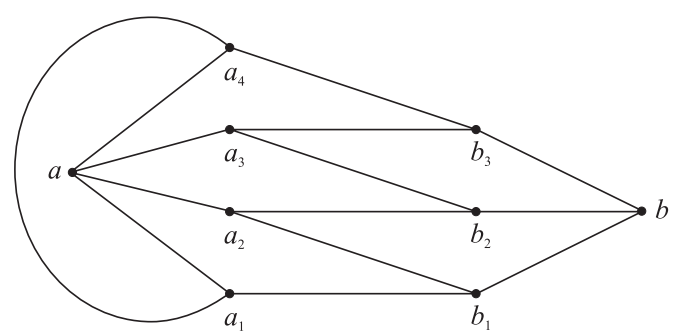

FIGURE 3. The graph $\mathbf{G}_{4}$.

- $\quad a_{i} \sim b_{i}$, for $1 \leq i \leq n-1$;

- $\quad a_{i} \sim b_{i-1}$, for $2 \leq i \leq n$;

- $\quad b \sim b_{i}$, for all $i$.

Figure 3 shows the graph $\mathbf{G}_{4}$. Define the connecting homomorphisms $\phi_{n}: \mathbf{G}_{n+1} \rightarrow$ $\mathbf{G}_{n}$ as follows. Relabel the vertices of $\mathbf{G}_{n+1}$ by $a^{\prime}, b^{\prime}, a_{1}^{\prime}, \ldots, a_{n+1}^{\prime}, b_{1}^{\prime}, \ldots, b_{n}^{\prime}$. Let $\phi_{n}$ be the map with $\phi_{n}\left(a^{\prime}\right)=a, \phi_{n}\left(b^{\prime}\right)=b, \phi_{n}\left(a_{i}^{\prime}\right)=a_{i}$ for $i \leq n, \phi_{n}\left(a_{n+1}^{\prime}\right)=a_{n}$, $\phi_{n}\left(b_{i}^{\prime}\right)=b_{i}$ for $i \leq n-1$, and $\phi_{n}\left(b_{n}^{\prime}\right)=b_{n-1}$, so $\phi_{n}$ is obviously a homomorphism.

If $\phi: \mathbf{G}_{n} \rightarrow \mathbf{K}_{3}$ is a homomorphism, then $\phi(a) \sim \phi(b)$, because otherwise $\phi(a)=$ $\phi(b)$, and therefore $\left\{a_{1}, \ldots, a_{n}, b_{1}, \ldots, b_{n-1}\right\}$ must be 2 -colourable, a contradiction. So $\mathbf{G}_{n}$ is pointwise nonseparable with respect to $\mathcal{R}\left(\mathbb{S}\left(\mathbf{K}_{3}\right)\right)$.

Let $A=\left\{a_{1}, \ldots, a_{n}\right\}$ and $B=\left\{b_{1}, \ldots b_{n-1}\right\}$. Note that $\mathbf{G}_{n}$ is 3-colourable; the map $f_{0}: \mathbf{G}_{n} \rightarrow \mathbf{K}_{3}$ with $f_{0}(a)=1, f_{0}\left(a_{1}\right)=2, f_{0}\left(A \backslash\left\{a_{1}\right\}\right)=3, f_{0}(B)=1$, $f_{0}(b)=2$ is a colouring. We show that every proper subgraph of $\mathbf{G}_{n}$ is freely 3-colourable, and therefore in $\mathcal{R}\left(\mathbb{S}\left(\mathbf{K}_{3}\right)\right)$. We first show that for every pair $x, y \in \mathbf{G}_{n}$ with $x \nsim y$ and $x \neq y$, there is a colouring of $\mathbf{G}_{n}$ where $x, y$ are coloured differently, and, provided $\{x, y\} \neq\{a, b\}$, there is also a colouring of $\mathbf{G}_{n}$ where $x, y$ are coloured the same. It then remains to show that if $\mathbf{H}$ is a proper subgraph of $\mathbf{G}$ with $a, b \in H$, there is a colouring of $\mathbf{H}$ where $a$ and $b$ are the same colour.

Let $x, y \in \mathbf{G}_{n}$ with $x \neq y$ and $x \nsim y$. We give a homomorphism $f: \mathbf{G}_{n} \rightarrow \mathbf{K}_{3}$ that colours $x, y$ differently.

Case A1. $x=a, y \in B$. At most one of $a_{1}, a_{n}$ is related to $y$. Without loss of generality, $a_{1} \nsim y$. Let $f$ be the map with $f(a)=1, f\left(a_{1}\right)=2, f\left(A \backslash\left\{a_{1}\right\}\right)=3$, $f(y)=2, f(B \backslash\{y\})=1, f(b)=3$.

Case A2. $x=a, y=b$. Let $f:=f_{0}$.

Case A3. $x, y \in A$. We have either $x \notin\left\{a_{1}, a_{n}\right\}$ or $y \notin\left\{a_{1}, a_{n}\right\}$. Suppose, without loss of generality, that $x \notin\left\{a_{1}, a_{n}\right\}$. Let $f$ be the map with $f(a)=1, f\left(a_{1}\right)=2$,

$$
f(x)= \begin{cases}3 & \text { if } y=a_{1}, \\ 2 & \text { otherwise }\end{cases}
$$

$f\left(A \backslash\left\{a_{1}, x\right\}\right)=3, f(B)=1, f(b)=2$. 
Case A4. $x \in A, y \in B$. Let $f:=f_{0}$.

Case A5. $x \in A, y=b$. If $x \neq a_{1}$, let $f:=f_{0}$. Otherwise, let $f=f_{1}$ be the map where $f(a)=1, f\left(a_{1}\right)=2, f\left(A \backslash\left\{a_{1}\right\}\right)=3, f(B)=1$, and $f(b)=3$.

Case A6. $x, y \in B$, say $x=b_{i}, y=b_{j}$ with $i<j$. Let $f$ be the map with $f(a)=1$,

$f(y)=2, f(B \backslash\{y\})=1, f(b)=3$.

$$
f\left(a_{k}\right)= \begin{cases}2 & \text { if } k<j, \\ 3 & \text { if } k \geq j,\end{cases}
$$

We now show that if $\{x, y\} \neq\{a, b\}$ there is a map $g: \mathbf{G}_{n} \rightarrow \mathbf{K}_{k}$ that colours $x, y$ the same. (Recall that $x, y$ are points such that $x \neq y$ and $x \nsim y$.)

Case B1. $x=a, y \in B$. Let $g:=f_{0}$.

Case B2. $x, y \in A$. Then $a_{1} \notin\{x, y\}$ or $a_{n} \notin\{x, y\}$. Suppose, without loss of generality, that $a_{1} \notin\{x, y\}$. Let $g:=f_{0}$.

Case B3. $x \in A, y \in B$, say $x=a_{i}$ and $y=b_{j}$. Either $i<j$ or $i>j+1$. If $i<j$, let $g$ be the map with $g(a)=1$,

$$
g\left(a_{k}\right)= \begin{cases}2 & \text { if } k \leq i \\ 3 & \text { if } k>i\end{cases}
$$

$g(y)=2, g(B \backslash\{y\})=1, g(b)=3$. If $i>j+1$, let $g$ be the map with $g(a)=1$,

$$
g\left(a_{k}\right)= \begin{cases}2 & \text { if } k<i, \\ 3 & \text { if } k \geq i,\end{cases}
$$

$g(y)=3, g(B \backslash\{y\})=1, g(b)=2$.

Case B4. $x \in A, y=b$. We have $x \neq a_{1}$ or $x \neq a_{n}$; without loss of generality, $x \neq a_{1}$. Let $g$ be the map $f_{1}$ from case A5.

Case B5. $x, y \in B$. Let $g:=f_{0}$.

We now show that for every proper subgraph $\mathbf{H}$ of $\mathbf{G}_{n}$ with $a, b \in H$, there is a colouring of $\mathbf{H}$ where $a$ and $b$ are coloured the same. Clearly it suffices to prove this for all the graphs $\mathbf{H}=\mathbf{G}_{n} \backslash\{z\}$, where $z \notin\{a, b\}$. If $z=a_{1}$ or $z=a_{n}$, then $\mathbf{G}_{n} \backslash\{z\}$ is bipartite. If $z=a_{i}$ or $z=b_{i}$ for some $i$, then $\mathbf{H} \backslash\{a, b\}$ is a tree, and therefore bipartite, so clearly there is a 3-colouring $f$ of $\mathbf{H}$ with $f(a)=f(b)$.

Thus every proper subgraph of $\mathbf{G}_{n}$ is freely 3-colourable, and therefore in $\mathcal{R}\left(\mathbb{S}\left(\mathbf{K}_{3}\right)\right)$. Define $\mathbf{G}:=\lim _{\longleftarrow}\left\{\mathbf{G}_{n} \mid n \in \mathbb{N}\right\}$. Then by Theorem 3.6,

$$
\mathbf{G} \in \operatorname{Mod}_{\mathrm{Bt}}\left(\operatorname{Th}_{\mathrm{uH}}\left(\mathbf{K}_{3}\right)\right) \backslash \mathcal{R}_{\mathrm{CT}}\left(\mathbb{S}\left(\mathbf{K}_{3}\right)\right),
$$

so $\mathcal{R}_{\mathrm{CT}}\left(\mathbb{S}\left(\mathbf{K}_{3}\right)\right)$ is not $\mathrm{uH}$ axiomatizable. 
LEMMA 4.4. Let $\mathbf{G}$ be a $k$-colourable graph. Then $\mathbf{G}$ is freely $(k+1)$-colourable.

PROOF. Label the vertices of $\mathbf{K}_{k}$ by $1,2, \ldots, k$. Let $a, b \in \mathbf{G}$ with $a \nsim b$ and $a \neq b$. To colour $a, b$ the same, let $\phi: \mathbf{G} \backslash\{a, b\} \rightarrow\{1, \ldots, k\}$ be a $k$-colouring of $\mathbf{G} \backslash\{a, b\}$. Then the map $\phi \cup\{(a, k+1),(b, k+1)\}$ is a $(k+1)$-colouring of $\mathbf{G}$. To colour $a, b$ differently, let $\phi: \mathbf{G} \backslash\{a\} \rightarrow\{1, \ldots, k\}$ be a $k$-colouring of $\mathbf{G} \backslash\{a\}$. Then the map $\phi \cup\{(a, k+1)\}$ is a $(k+1)$-colouring of $\mathbf{G}$.

THEOREM 4.5. Let $k \geq 3$, and let $\mathbf{K}_{k}$ be the complete graph on $k$ vertices. Then $\mathcal{R}_{\mathrm{CT}}\left(\mathbb{S}\left(\mathbf{K}_{k}\right)\right)$ is not $u H$ axiomatizable.

PROOF. The proof is by induction. Let $k \geq 3$. The inductive hypothesis is that there is an inverse system of graphs $\left\{\mathbf{G}_{n}^{k} \mid n \in \mathbb{N}\right\}$ with connecting homomorphisms $\phi_{n}^{k}: \mathbf{G}_{n+1}^{k} \rightarrow \mathbf{G}_{n}^{k}$ such that:

(1) there exist elements $a^{k}, b^{k}$ of the inverse limit such that, for each $n$, we have $a_{n}^{k} \nsim b_{n}^{k}$ but, for every homomorphism $\psi: \mathbf{G}_{n}^{k} \rightarrow \mathbf{K}_{k}$, we have $\psi\left(a_{n}^{k}\right) \sim \psi\left(b_{n}^{k}\right)$;

(2) $G_{n}^{k}$ is $k$-colourable;

(3) every proper subgraph of $\mathbf{G}_{n}^{k}$ is in $\mathcal{R}\left(\mathbb{S}\left(\mathbf{K}_{k}\right)\right)$.

By the proof of Lemma 4.3, this is true for $k=3$. Now assume it is true for some $k \geq 3$. We construct an inverse system $\left\{\mathbf{G}_{n}^{k+1} \mid n \in \mathbb{N}\right\}$ satisfying (1)-(3). For each $n$, let $\mathbf{G}_{n}^{k+1}$ be the graph with underlying set $\mathbf{G}_{n}^{k} \cup\left\{y_{n}\right\}$ and the relation that contains all the edges from $\mathbf{G}_{n}^{k}$ and the additional edges $\left\{x, y_{n}\right\}$ for each $x \in G_{n}^{k}$. Let $\phi_{n}^{k+1}: \mathbf{G}_{n+1}^{k+1} \rightarrow \mathbf{G}_{n}^{k+1}$ be the map $\phi_{n}^{k} \cup\left\{\left(y_{n+1}, y_{n}\right)\right\}$, so $\phi_{n}^{k+1}$ is clearly a homomorphism, since $\phi_{n}^{k}$ is.

To show that (1) holds for $G_{n}^{k+1}$, choose $a^{k+1}:=a^{k}$ and $b^{k+1}:=b^{k}$, and suppose that $\psi: \mathbf{G}_{n}^{k+1} \rightarrow \mathbf{K}_{k+1}$ is a homomorphism. Since $y_{n} \sim x_{n}$ for each $x_{n} \in G_{n}^{k}$, there exists $i \in K_{k+1}$ such that $\psi\left(y_{n}\right)=i$ and $\psi\left(G_{n}^{k}\right)=\psi\left(G_{n}^{k+1} \backslash\left\{y_{n}\right\}\right) \subseteq K_{k+1} \backslash\{i\}$. Without loss of generality we can assume that $i=k+1$, so that $\psi \uparrow_{G_{n}^{k}}\left(G_{n}^{k}\right) \subseteq K_{k}$. Therefore, since (1) holds for $k$, we have $\psi\left(a_{n}^{k+1}\right) \sim \psi\left(b_{n}^{k+1}\right)$.

Since $G_{n}^{k}$ is $k$-colourable, it is obvious that $G_{n}^{k+1}$ is $(k+1)$-colourable, so (2) holds.

To prove (3), let $\mathbf{H}$ be a proper subgraph of $\mathbf{G}_{n}^{k+1}$. Since $\mathbf{G}_{n}^{k+1}$ is $(k+1)$-colourable, $\mathbf{H}$ is also $(k+1)$-colourable. We now show that $\mathbf{H}$ is freely $(k+1)$-colourable. If $y_{n} \notin H$, then $H \subseteq G_{n}^{k}$, so $\mathbf{H}$ is $k$-colourable and therefore freely $(k+1)$-colourable by Lemma 4.4. Now assume that $y_{n} \in H$, and let $u_{n}, v_{n} \in \mathbf{H}$ with $u_{n} \neq v_{n}$ and $u_{n} \nsim v_{n}$. Then $u_{n}, v_{n} \neq y_{n}$. Since $\mathbf{H} \backslash\left\{y_{n}\right\}$ is a proper subgraph of $\mathbf{G}_{n}^{k}$ and (3) holds for $\mathbf{G}_{n}^{k}$, there exist homomorphisms $\phi_{1}^{\prime}: \mathbf{H} \backslash\left\{y_{n}\right\} \rightarrow \mathbf{K}_{k}$ and $\phi_{2}^{\prime}: \mathbf{H} \backslash\left\{y_{n}\right\} \rightarrow \mathbf{K}_{k}$ such that $\phi_{1}^{\prime}\left(u_{n}\right)=\phi_{1}^{\prime}\left(v_{n}\right)$ and $\phi_{2}^{\prime}\left(u_{n}\right) \neq \phi_{2}^{\prime}\left(v_{n}\right)$. The maps $\phi_{1}: \mathbf{H} \rightarrow \mathbf{K}_{k+1}$ and $\phi_{2}: \mathbf{H} \rightarrow \mathbf{K}_{k+1}$ given by

$$
\phi_{1}=\phi_{1}^{\prime} \cup\left\{\left(y_{n}, k+1\right)\right\} \quad \text { and } \quad \phi_{2}=\phi_{2}^{\prime} \cup\left\{\left(y_{n}, k+1\right)\right\}
$$

are homomorphisms and we have $\phi_{1}\left(u_{n}\right)=\phi_{1}\left(v_{n}\right)$ and $\phi_{2}\left(u_{n}\right) \neq \phi_{2}\left(v_{n}\right)$. Thus (3) holds for $k+1$.

Therefore by induction, for each $k \geq 3$, there exists an inverse system satisfying (1) and (3), so by Theorem 3.6, $\mathcal{R}_{\mathrm{CT}}\left(\mathbb{S}\left(\mathbf{K}_{k}\right)\right)$ is not $\mathrm{uH}$ axiomatizable. 


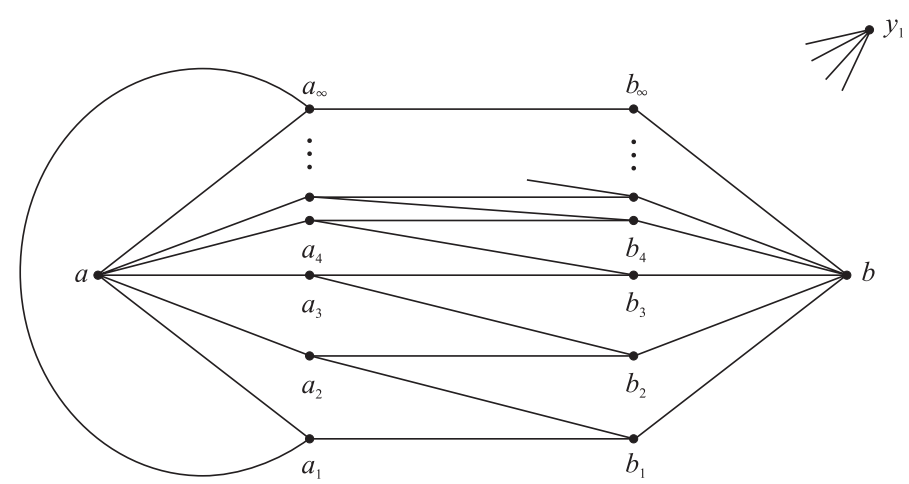

FIgURE 4. The inverse limit constructed in the proof of Theorem 4.5 , where $k=4$. The vertex $y_{1}$ is related to every other vertex, but these edges are not shown. In general, for $k=n$, there are vertices $y_{1}, \ldots, y_{n-3}$ that are related to every other vertex.

We will again use a theorem from [5] to show that $\mathcal{R}_{\mathrm{CT}}\left(\mathbb{S}\left(\mathbf{K}_{k}\right)\right)$ is not first-order axiomatizable. We give the simplified version of the theorem that is applicable in the case of graphs. We first require some basic facts about compactifications. Let $X$ be a set and $\lambda \in \mathbb{N}$. A $\lambda$-compactification of $X$ is an idempotent map $f: X \rightarrow X$ such that $|f(X)| \leq \lambda$. A $\lambda$-compactification $f$ of $X$ is a unary operation on $X$, and we write $\langle X ; \sim, f\rangle$ to denote the structure consisting of the set $X$ with the graph edge relation $\sim$ and the operation $f$. If $f: X \rightarrow X$ is a $\lambda$-compactification, the $\lambda$-compactification topology induced by $f$ is the topology where $U$ is open if and only if, for each element $x$ of $f(X)$ in $U$, the set $U$ contains cofinitely many elements of $f^{-1}(x)$ (in particular, $U$ is open if $f(X) \cap U$ is empty). The $\lambda$-compactification topology is always Boolean. Also, the property that $f$ is a $\lambda$-compactification of $X$ can be expressed by universal sentences, so if $f$ is a $\lambda$-compactification of $X$ and $\langle Y ; g\rangle$ satisfies the universal theory of $\langle X ; f\rangle$, then $g$ is a $\lambda$-compactification of $Y$.

Theorem 4.6 [5, Theorem 5.3]. Let $\mathcal{U}$ be a $u H$ class and $\lambda \in \mathbb{N}$. Suppose that $\mathbf{X} \in \mathcal{U}_{\mathrm{Bt}} \backslash \mathcal{R}_{\mathrm{CT}}\left(\mathcal{U}_{\mathrm{fin}}\right)$ is such that:

(1) the topology on $\mathbf{X}$ is the $\lambda$-compactification topology; and

(2) for every model $\langle Y ; \bowtie, g\rangle$ of the universal theory of $\langle X ; \sim, f\rangle$, the relation $\bowtie$ is closed in $Y^{2}$, where $Y$ has the $\lambda$-compactification topology induced by $g$.

Then $\mathcal{R}_{\mathrm{CT}}\left(\mathcal{U}_{\text {fin }}\right)$ is not first-order axiomatizable.

TheOREM 4.7. Let $k \geq 3$ and let $\mathbf{K}_{k}$ be the complete graph on $k$ vertices. Then $\mathcal{R}_{\mathrm{CT}}\left(\mathbb{S}\left(\mathbf{K}_{k}\right)\right)$ is not first-order axiomatizable.

Proof. Let $\mathbf{X}:=\lim \left\{\mathbf{G}_{n}^{k}: n \in \mathbb{N}\right\}$ be the inverse limit constructed in Theorem 4.5. The case $k=4$ is shown in Figure 4 . The topological graph $\mathbf{X}$ has underlying set

$$
\left\{a_{1}, \ldots, a_{\infty}, b_{1}, \ldots, b_{\infty}, a, b, y_{1}, \ldots, y_{k-3}\right\}
$$


and relation $\sim$ such that:

- $\quad a \sim a_{i}$, for all $i$;

- $\quad a_{1} \sim a_{\infty}$

- $\quad a_{i} \sim b_{i}$, for all $i$

- $\quad a_{i} \sim b_{i-1}$, for $2 \leq i<\infty$;

- $\quad b \sim b_{i}$, for all $i$;

- $\quad y_{i} \sim x$, for all $i$ and all $x \in X \backslash\left\{y_{i}\right\}$

The topology on $\mathbf{X}$ is the $(k+1)$-compactification topology induced by the map $f: X \rightarrow X$ given by $f(a)=a, f(b)=b, f\left(a_{i}\right)=a_{\infty}$ for all $i, f\left(b_{i}\right)=b_{\infty}$ for all $i$, and $f\left(y_{i}\right)=y_{i}$ for all $i$.

Now let $\langle Y ; \bowtie, g\rangle$ be a model of the universal theory of $\langle X ; \sim, f\rangle$, so that $g$ is a $(k+1)$-compactification of $Y$ and $\langle Y ; \bowtie, g\rangle$ satisfies the following sentences.

(1) $\quad(\forall x, y) x \bowtie y \longrightarrow g(x) \bowtie g(y)$ or $g(x) \approx g(y)$;

(2) $\quad(\forall x, y, z, w)(x \bowtie y \& g(x) \approx g(y) \& z \bowtie w \& g(z) \approx g(w)) \longrightarrow$ $\{x, y\} \approx\{z, w\}$

(3) $\left(\forall x, z_{1}, \ldots, z_{k+1}\right)\left(x \bowtie z_{1} \& x \bowtie z_{2} \& \cdots \& x \bowtie z_{k+1}\right) \longrightarrow$ $\left(g(x) \approx x\right.$ or $z_{i} \approx z_{j}$ for some $i, j \leq k$ with $\left.i \neq j\right)$.

Note that (2) says that there is at most one set $\{x, y\}$ such that $x \bowtie y$ and $g(x)=g(y)$, and (3) says that if $g(x) \neq x$ then $x$ is related to at most $k$ other vertices.

To show that $\bowtie$ is closed in $Y^{2}$, let $u, v \in \mathbf{Y}$ with $u \bowtie v$. We must find an open set $U \ni(u, v)$ such that $U \cap \bowtie=\varnothing$. We need the following observation, which follows from the fact that $g$ is idempotent:

(*) if $u \in g(Y)$, then $g^{-1}(u) \cap g(Y)=\{u\}$. Hence, if $u \in g(Y)$ and $F$ is finite, then $g^{-1}(u) \backslash F$ is open.

Case 1. $u, v \notin g(Y)$. Then $\{u\} \times\{v\}$ is open and disjoint from $\bowtie$.

Case 2. $u, v \in g(Y)$.

Case 2a. $u=v$. Let $W$ be the set of all pairs $(x, y)$ such that $x \bowtie y$ and $g(x)=g(y)$. Clearly $\left(g^{-1}(u) \times g^{-1}(u)\right) \backslash W$ contains $(u, v)$ and is disjoint from $\bowtie$. Also, by (2), we have $|W| \leq 2$, so $\left(g^{-1}(u) \times g^{-1}(u)\right) \backslash W$ is open by $(*)$.

Case $2 b . \quad u \neq v$. The set $g^{-1}(u) \times g^{-1}(v)$ contains $(u, v)$ and by $(*)$ it is open. Also, by (1) it is disjoint from $\bowtie$.

Case 3. $u \in g(Y)$ and $v \notin g(Y)$. Then $\{v\}$ is open. Let $X=\{x \mid x \bowtie v\}$. Now $g(v) \neq v$, so by (3), we have $|X| \leq k$. Therefore by $(*)$, the set $g^{-1}(u) \backslash X$ is open. So $\left(g^{-1}(u) \backslash X\right) \times\{v\}$ is open, contains $(u, v)$, and is disjoint from $\bowtie$.

We have shown that $\bowtie$ is closed. Hence $\mathcal{R}_{\mathrm{CT}}\left(\mathbb{S}\left(\mathbf{K}_{k}\right)\right)$ is not first-order axiomatizable by Theorem 4.6.

\section{References}

[1] S. Burris and H. P. Sankappanavar, 'A course in universal algebra', online at http://www.math.uwaterloo.ca/ snburris/htdocs/ualg.html, originally published in 1981. 
[2] X. Caicedo, 'Finitely axiomatisable quasivarieties of graphs', Algebra Universalis 34 (1995), 314-321.

[3] D. M. Clark, B. A. Davey, R. S. Freese and M. Jackson, 'Standard topological algebras: syntactic and principal congruences and profiniteness', Algebra Universalis 52 (2004), 343-376.

[4] D. M. Clark, B. A. Davey, M. Haviar, J. G. Pitkethly and M. R. Talukder, 'Standard topological quasi-varieties', Houston J. Math. 4 (2003), 859-887.

[5] D. M. Clark, B. A. Davey, M. G. Jackson and J. G. Pitkethly, 'The axiomatizability of topological prevarieties', Adv. Math. 218 (2008), 1604-1653.

[6] B. A. Davey, 'Natural dualities for structures', Acta Univ. M. Belii Ser. Math. 13 (2006), 3-28.

[7] B. A. Davey and M. R. Talukder, 'Dual categories for endodualisable Heyting algebras: optimization and axiomatization', Algebra Universalis 53 (2005), 331-355.

[8] C. Edwards, 'Standardness of small topological structures', MSc Thesis, La Trobe University, 2005.

[9] T. Feder and M. Y. Vardi, 'The computational structure of monotone monadic SNP and constraint satisfaction: a study through Datalog and group theory', SIAM J. Comput. 28 (1998), 57-104.

[10] P. Hell and J. Nešetřil, 'The core of a graph', Discrete Math. 109 (1992), 117-126.

[11] I. Hodkinson and Y. Venema, 'Canonical varieties with no canonical axiomatisation', Trans. Amer. Math. Soc. 357 (2005), 4579-4605.

[12] M. Jackson, 'Residual bounds for compact totally disconnected algebras', Houston J. Math. 34 (2008), 33-67.

[13] J. Nešetřil and A. Pultr, 'On classes of relations and graphs determined by subobjects and factorobjects', Discrete Math. 22 (1978), 287-300.

[14] A. Stralka, 'A partially ordered space which is not a Priestley space', Semigroup Forum 20 (1980), 293-297.

[15] B. Trotta, 'Residual properties of pre-bipartite digraphs', Algebra Universalis, to appear.

[16] B. Trotta, 'Residual properties of reflexive anti-symmetric digraphs', Houston J. Math., to appear.

BELINDA TROTTA, Department of Mathematics and Statistics, La Trobe University, Bundoora, Victoria 3086, Australia

e-mail: B.Trotta@mmassociates.com.au 